James Madison University JMU Scholarly Commons

Libraries

Libraries \& Educational Technologies

January 2011

\title{
EBSCO Computers and Applied Sciences Complete and ProQuest Computer Science Collection
}

\section{Jody C. Fagan}

James Madison University, faganjc@jmu.edu

D.J. Gaines

Follow this and additional works at: http://commons.lib.jmu.edu/letfspubs

Part of the Library and Information Science Commons

\section{Recommended Citation}

Fagan, Jody C. and Gaines, D. J., "EBSCO Computers and Applied Sciences Complete and ProQuest Computer Science Collection" (2011). Libraries. Paper 41.

http://commons.lib.jmu.edu/letfspubs/41

This Article is brought to you for free and open access by the Libraries \& Educational Technologies at JMU Scholarly Commons. It has been accepted for inclusion in Libraries by an authorized administrator of JMU Scholarly Commons. For more information, please contact dc_admin@jmu.edu. 


\section{\$ EBSCO Computers and Applied Sciences Complete and ProQuest Computer Science Collection}

\author{
EBSCO Composite Score: \\ ProQuest Composite Score:
}

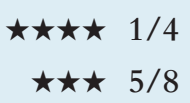

Reviewed by: Jody Condit Fagan

Content Interfaces Coordinator and Associate Professor

Carrier Library

James Madison University

$<$ faganjc@jmu.edu>

David J. Gaines

<atrosbaka@gmail.com>

\begin{abstract}
This article provides a comparative review of EBSCO Computers and Applied Sciences Complete and ProQuest Computer Science Collection looking at the strengths and weaknesses of each product. Both products use the vendor's standard search interface. With EBSCO, a major upgrade came in Fall 2010; with ProQuest, an even more dramatic upgrade came in Spring 2011 that included merging CSA and ProQuest products and interfaces. Both platforms offer interface customization options via an administrator interface which can affect the search experience. Both products index the core publications of the field, including those from IEEE and the Association for Computing Machinery (ACM). However, differences in coverage are noted.
\end{abstract}

\section{Pricing Options}

For EBSCO's Computers and Applied Sciences Complete, pricing factors include FTE, existing EBSCO subscriptions and purchases, and consortial and other group buying agreements. EBSCO will provide custom quotes for specific libraries. Pricing for ProQuest's Computer Science collection is also based on a range of factors including type and size of institution and the library's other ProQuest subscriptions and purchases. Consortial pricing is available; additional information is available from ProQuest.

\section{Product Description}

EBSCO's Computers and Applied Sciences Complete (CASC) covers the 1960 s to present, with at least some titles indexed as far back as 1956. Indexing and abstracts are provided for over 2,000 academic journals, professional publications, and other reference sources, with full text for more than 1,000 titles. As of this review, about 1,420 of the journals are listed as peer reviewed by EBSCO. According to EBSCO, the subject coverage of CASC focuses on research and development, engineering challenges, and business and social implications of technologies. EBSCO also offers Computer Science Index, which appears to be a subset of CASC focusing on academic journals and scholarly materials.

ProQuest's Computer Science Collection (CSC) is an amalgamation of three components: Computer and Information Systems Abstracts, ProQuest Computer Science Journals, and ProQuest Deep Indexing: Computer Science. Together, these elements cover 1981 to present. Three thousand international journals, conference proceedings, reports, patents, books, and press releases are indexed, and 600 of the journals, including peer reviewed and trade journals, are available in full text. As of this review, ProQuest states about 960 journals are peer reviewed. The last element mentioned, Deep Indexing, adds indexing of tables and figures designed to increase search precision. Subject coverage includes both theoretical and practical research. Customers

\section{"At a Glance" Comparative Review Scores}

The maximum number of stars in each category is 5 .

\section{EBSCO Computers and Applied Sciences Complete}

\section{ProQuest Computer} Science Collection

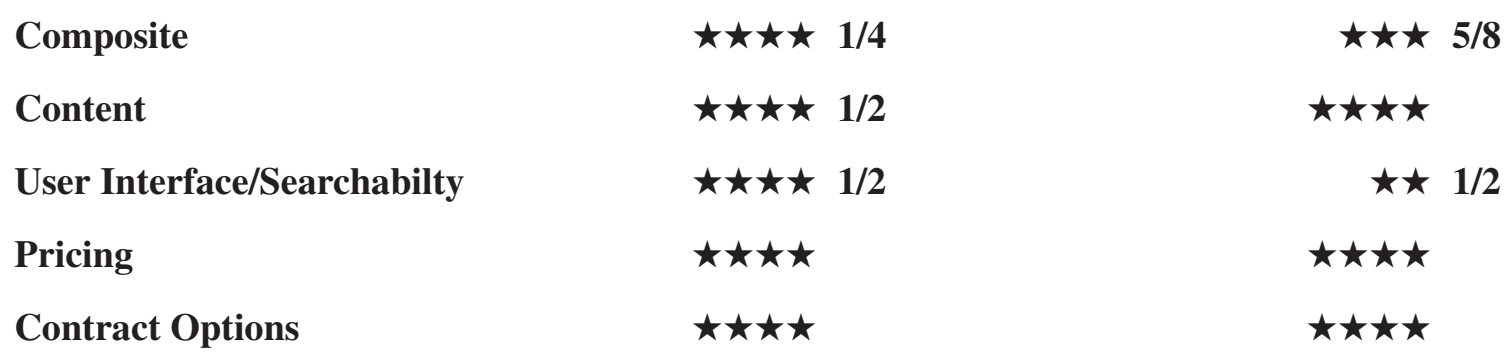


can subscribe to just Computer and Information Systems Abstracts (ProQuest Deep Indexing: Computer Science is included) or the ProQuest Computer Science Collection (Computer and Information Systems Abstracts, ProQuest Computer Science Journals, ProQuest Deep Indexing: Computer Science) which is the product reviewed here. In either case, log in links can be setup to any individual components.

\section{Critical Evaluation}

One way to examine the content of the two products is to look at vendor-provided title lists. EBSCO contains about 2,250 periodical titles. Some of the length of EBSCO's list appears to be due to its inclusion of applied topics. For example, it includes titles such as Ground Water (Wiley-Blackwell), Recycling Today (GIE Media), and School Library Media Monthly (Greenwood). The ProQuest Computer Science Collection contains about 1,790 periodicals, plus selective coverage of 3,336 publications from diverse applied science areas, including Journal of Gambling Studies. Both products index the core publications of the field, including those from IEEE and the Association for Computing Machinery (ACM), but EBSCO offers full text for the latter, including the Communications of the ACM since 1965.

Because differences in the vendor lists make further comparison challenging, the authors attempted to conduct keyword and controlled vocabulary searches in both products to compare results sets. Unfortunately, this proved useless for the purpose because ProQuest's result numbers fluctuated wildly during the testing period. For example, on April 14, 2011, a search on "artificial intelligence" limited to 20062010 retrieved 45,862 results. On May 13, the same search retrieved 17,215 results; on May 14, 43,784; and on June7, 46,864. When confronted with these numbers, ProQuest said they suspected a "content loading issue," which could be the result of the database being updated or documents being corrected that needed to be removed.

The search differences did illuminate a major difference in approach to search between the two systems. All searches, including those limited to the subject field or limited to the past five years, returned many more results in ProQuest's product than in EBSCO's. For example, a search on "artificial intelligence" in EBSCO limited to 2006-2010 found 8,699 results compared with ProQuest's 46,864. This appears to be partially related to the inclusion of more diverse subject areas in ProQuest, but mostly because of differences in the search algorithms. ProQuest, therefore, may be favored by students and other users accustomed to Google-like searching, whereas EBSCO's more controlled search may be more favorable to expert searchers.

\section{INDEXES}

EBSCO has 14 indexes located in a hidden menu (under More in the header). While some are of dubious value (for example, the Products and Reviews index seems to list only individual books), others may help users select appropriate terms. For example, the People and Company Entity index differentiates between similar names. Using EBSCO's indexes may produce different numbers of results than field searching in the same field, because the indexes are phrase-indexed. For example, using the Subject index will perform an exact phrase search on the given subject, while searching the same words using the Subject field limit will perform a keyword search on both subject and geographic terms.

Whereas the previous version of ProQuest/CSA Computer and Information Systems Abstracts had three easy-to-browse indexes (Author, Journal Name, and Publication Type), ProQuest's new Computer Sci- ence Collection appears to employ a spellcheck-style index for some fields. It is impossible to tell which fields will invoke this feature; Subject and Publisher do not, but Document Title and Publication Title do. This feature is somewhat messy and hard to interpret; if one is looking for the Communications of the ACM and chooses Publication Title, then types in "communica," one of the entries that appears is "communicatep did you say communicate?" and if one chooses "communications of the acm" one receives a different number of results (about 400 items difference) than if one chooses "acm communications" (which appears earliest in the typing process). In addition, if one chooses the latter entry, one is asked, "Did you mean pub(am communications)" which itself results in 0 results. Clearly, this feature is still in development.

ProQuest also offers the ability to browse some indexes from the advanced search screen but, again, it is impossible to tell which fields will reveal this feature. Publication Title offers Look up Publications, but Tags does not offer a similar feature. Using these Look Up options causes a pop-up panel to display with a searchable list. When Communications of the ACM was selected, it found fewer results than either of the spellcheck attempts mentioned earlier. This appears to be because ProQuest performs an exact phrase search when using the Look Up indexes.

\section{SEARCHABILITY}

Both products use the vendor's standard search interface. With EBSCO, a major upgrade came in Fall 2010; with ProQuest, an even more dramatic upgrade came in Spring 2011 that included merging CSA and ProQuest products and interfaces. Both platforms offer interface customization options via an administrator interface which can affect the search experience. Both products can be set to default to a basic interface with one search box, or to an advanced interface with multiple search boxes and fields. The advanced interface will be used to compare the two products' searchability in this review.

On EBSCO's advanced search, the most relevant limits for Computer Science are full text, peer reviewed, and date limits. Publication Type allows one to limit to academic journal, periodical, reference book, and trade publication; Document Type allows limiting by numerous options such as article, book chapter, case study, or editorial. The number of pages limit is a nice addition.

ProQuest's advanced search limits include both scholarly journals and peer reviewed journals, date limits, source type, and document type. Source Type includes books, conference papers, dissertations, government and official publications, other sources, reports, and scholarly journals; Document Type contains similar options to EBSCO, although there is currently some messiness such as "Electronic Journal Article DE:" and both "Journal_Articles" and "Journal Article." An annoyance is that when one uses a field search, the field codes are entered into the search box and persist across screens. Thus, if one does a Subject search, then wants to modify the search to a Keyword search, one must manually delete the sub( ) codes from around the terms. This also happens if one clicks on a hyperlinked subject heading from a result. A company representative explained: "This is part of our design. The idea is that we want to be as transparent with our searches and what the search engine is doing as possible. Keeping these codes in informs the search engine where to search but it also informs the user what is occurring. This helps end user researchers but also search experts like librarians who may be asked to provide guidance on search query construction and syntax." (ProQuest, personal communication, 2011) 


\section{ProQuest Computer Science Collection Review Scores Composite: $\star \star \star 5 / 8$}

The maximum number of stars in each category is 5 .

\section{Content:}

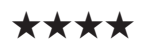

The indexing coverage is comparable to EBSCO, but EBSCO seems to offer more core full text. The diversity of selective coverage is a nice way to supplement core titles.

\section{User Interface/Searchability: $\quad \star \star 1 / 2$}

ProQuest's new platform shows promise, but struggles in its execution.

\section{Pricing:}

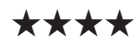

A variety of options for individual libraries and consortia.

\section{Contract Options:}

Meets the industry standard for a commercial publisher.

\begin{abstract}
EBSCO's results screen offers limiters and facets on the left-hand side. The top group of limiters includes check boxes for full text, references available, peer reviewed journals, and date. The facets include source types, subject, and publication. Document type could be a useful addition to these facets. It would be nice if the number of items for each facet were listed, to help prevent going down too narrow a path. Although the facets reset themselves when a new search is performed, they stay open if one opens them. The results area shows citation information, subject terms, thumbnails for article images, and links to full text or the link resolver.
\end{abstract}

ProQuest's results screen offers suggested subjects at the top; these could be useful to students or researchers exploring a new topic area. Facets are displayed in the right-hand column, with the number of items in each category. The number of items is useful for understanding the dimensions of the results set; however, only the first facet (source type) is expanded by default. The other facets are publication title, document type, keyword, subject, classification, location, language, and date. The document type seems more useful than the source type, for example, Journal Article, which is a document type, usually has more results than Scholarly Journals. While the other facets seem like they could be useful to some researchers, Location did not appear useful to any of the test searches, only having one or two items for each location listed. The facets closed and reset themselves after each new search, meaning that if one found a particular facet useful, one was required to keep re-opening it and selecting it. Similar to EBSCO, the results area shows a brief citation, thumbnails for article figures and tables, and links to full text or the link resolver. ProQuest also offers an explicit Search Within feature via a hyperlink.

On the record view, both products offer the ability to search for similar items; however, ProQuest's currently took too long to produce results or would list only duplicate items (ProQuest says this will be addressed in a coming release). EBSCO's similar items seemed relevant to the original article. EBSCO also offered the ability to list the article's references from the full record view.
A nice extra in EBSCO's product is the ability to perform Cited References searching, which can be used to find articles that have cited a previous article, similar to Science Citation Index. While this is a useful feature, it is naturally limited to just publications indexed in Computers and Applied Sciences Complete.

ProQuest's new platform is currently rife with technical glitches, some of which were mentioned earlier in this review. The company confirmed the platform is launched and customers are currently migrating to it; they have additional enhancement releases scheduled for July, October, and December of 2011. Some glitches found by this reviewer in May were fixed with an early June release, showing the company is making progress. Still, problems remain with search sessions timing out for no apparent reason, odd results in the spellchecker, and database content issues. For example, this reviewer found a recent article from Communications of the ACM in EBSCO that could

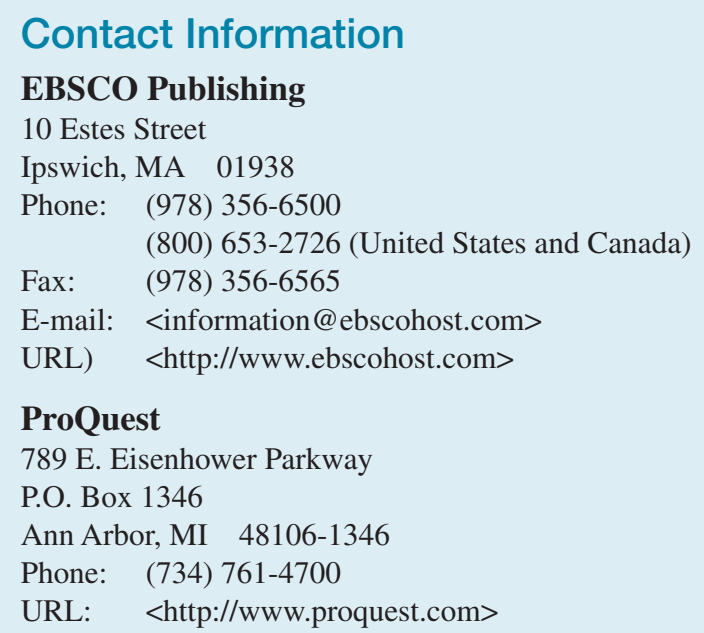

\section{ProQuest}

789 E. Eisenhower Parkway

P.O. Box 1346

Ann Arbor, MI 48106-1346

Phone: (734) 761-4700

URL: <http://www.proquest.com> 


\section{EBSCO Computers and Applied Sciences Complete Review Scores Composite: $\star \star \star \star \star 1 / 4$}

The maximum number of stars in each category is 5 .

\section{Content: $\quad \star \star \star \star 1 / 2$}

EBSCO has thoroughly covered the field and offers a good selection of full text. Results are available for very recent items.

\section{User Interface/Searchability: $\quad \star \star \star \star 1 / 2$ \\ EBSCO's standard platform provides a full suite of tools.}

\section{Pricing:}

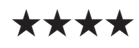

A variety of options for individual libraries and consortia.

\section{Contract Options:}

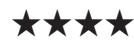

Meets the industry standard for a commercial publisher.

not be found in ProQuest; company representatives confirmed the issue and are looking into it.

\section{Contract Provisions}

EBSCO's standard contract includes use of the product for interlibrary loan, library reserves, and course packs. No commercial uses are permitted, nor can substantial portions be downloaded, copied, or printed. The terms of use require the author and copyright to not be removed or altered. Institutionally-affiliated users can access the database remotely, and any user can access the database if physically located at the institution.

ProQuest's contract is similar and allows for interlibrary loan and scholarly sharing. Electronic course reserves must employ durable links rather than a locally saved copy. Use is permitted for educational, scientific, or research purposes only, and downloading, copying, and printing must comply with fair use. For public libraries, library staff, residents of the library's geographic area, and walk-in patrons on site are considered authorized users; for academic institutions, currently enrolled students, faculty, staff, visiting scholars, and on-site walk-in patrons are authorized.

\section{Authentication}

EBSCOhost offers several different methods of authentication for users, including IP address, patterned IDs, patron ID files, referring URL, user name and password, cookie authentication, Athens, Shibboleth, and HTTP. The minimum browser requirements are Microsoft Internet Explorer 6.0 or later and Firefox 2.0 or later for Windows, and Safari 2.0x or Firefox 2.0 or later (build 412+) for Macintosh.
ProQuest offers IP authentication, Athens, Shibboleth, referring URL, barcode, institutional user name and password, and personal user name and password. ProQuest recommends Internet Explorer 7.0 and 8.0, Firefox 3.5 or higher, Google Chrome, or Safari 4. IE 9 and Firefox version 4.0 are not recommended. While earlier versions of browsers such as Internet Explorer 6.0 may function, noncritical problems using these browsers will not be supported by ProQuest.

\section{Author's References}

EBSCOhost. Computers and Applied Sciences Complete. <http:// www.ebscohost.com/academic/computers-applied-sciences-complete $>$. Accessed May 14, 2011.

EBSCOhost. Computer Science Index. <http://www.ebscohost.com/ academic/computer-science-index >. Accessed May 14, 2011.

ProQuest. ProQuest Computer Science Collection. <http://www. proquest.com/en-US/catalogs/databases/detail/computer_science. shtml>. Accessed May 14, 2011.

\section{About the Author}

Jody Condit Fagan is Associate Professor and Director of Scholarly Content Systems at James Madison University, Harrisonburg, VA. She has published numerous articles and two books, including Web Project Management in Academic Libraries with colleague Jennifer A. Keach (Chandos, 2009) and Comic Book Collections for Libraries with Bryan D. Fagan (Libraries Unlimited, 2011).

David J. Gaines is currently studying librarianship and volunteering at James Madison University Libraries. 